\title{
Synthesis, NMR and X-ray Studies of Iridium Dihydride C,N and N,P Ligand Complexes
}

\author{
Stefan Gruber*
}

Department of Chemistry, University of Basel, St. Johanns-Ring 19, 4056 Basel, Switzerland

E-mail: st_gr@gmx.ch

Present Address: University of Oxford, Chemistry Research Laboratory, 12 Mansfield Road, OXI 3TA, Oxford, United Kingdom

\section{Supplementary Information}

\section{Characterization}
A. Characterization and labeling of complexes 5, 6a, and $\mathbf{6 b}$
B. X-ray Crystallography
C. ${ }^{1} \mathrm{H},{ }^{31} \mathrm{P}$ and ${ }^{13} \mathrm{C}$ NMR spectra for complexes $5,6 \mathbf{a}$, and $\mathbf{6 b}$ 
Table S1. ${ }^{1} \mathrm{H}$ - and ${ }^{13} \mathrm{C}-\mathrm{NMR}$ data for the cation of salt 5 in $\mathrm{CD}_{2} \mathrm{Cl}_{2}$ at $295 \mathrm{~K}$.

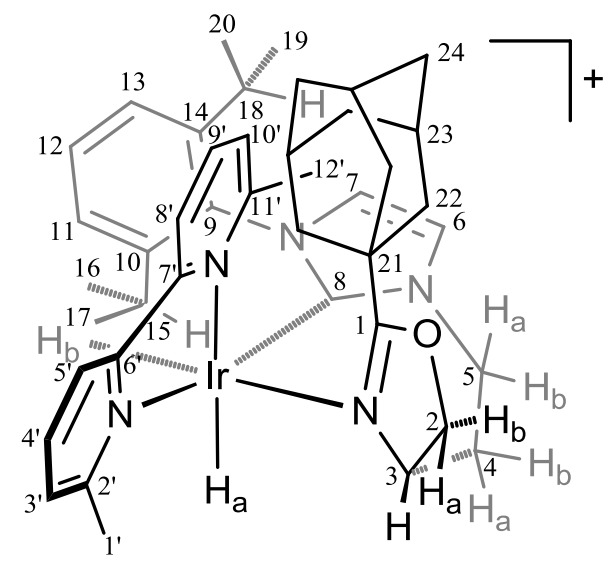

\begin{tabular}{|c|c|c|}
\hline Position & $\begin{array}{c}\delta\left({ }^{1} \mathrm{H}\right) \\
{[\mathrm{ppm}]}\end{array}$ & $\begin{array}{l}\delta\left({ }^{13} \mathrm{C}\right) \\
{[\mathrm{ppm}]}\end{array}$ \\
\hline $\mathrm{Ir}-\mathrm{H}_{\mathrm{a}}$ & -21.86 & - \\
\hline $\mathrm{Ir}-\mathrm{H}_{\mathrm{b}}$ & -28.63 & - \\
\hline 1 & - & 180.1 \\
\hline $2 a$ & 4.15 & 72.7 \\
\hline $2 b$ & 4.07 & 72.7 \\
\hline 3 & 4.84 & 73.2 \\
\hline $4 a$ & 2.24 & 36.4 \\
\hline $4 b$ & 1.79 & 36.4 \\
\hline $5 a$ & 4.83 & 46.6 \\
\hline $5 b$ & 4.02 & 46.6 \\
\hline 6 & 7.10 & 121.1 \\
\hline 7 & 6.91 & 123.8 \\
\hline 8 & - & 158.1 \\
\hline 9 & - & 138.5 \\
\hline 10 & - & 146.4 \\
\hline 11 & 7.14 & 124.6 \\
\hline 12 & 7.29 & 129.6 \\
\hline 13 & 6.98 & 123.9 \\
\hline 14 & - & 145.3 \\
\hline 15 & 2.26 & 29.0 \\
\hline 16 & 1.06 & 25.3 \\
\hline 17 & 1.32 & 22.9 \\
\hline 18 & 2.39 & 29.0 \\
\hline 19 & 0.96 & 24.7 \\
\hline 20 & 0.36 & 21.4 \\
\hline 21 & - & 36.8 \\
\hline 22 & $1.35 / 1.11$ & 38.7 \\
\hline 23 & 1.41 & 27.7 \\
\hline 24 & $1.39 / 1.08$ & 36.1 \\
\hline
\end{tabular}


Table S2. ${ }^{1} \mathrm{H}$ - and ${ }^{13} \mathrm{C}-\mathrm{NMR}$ data for the major species $(6 \mathrm{a})$ of salt 6 in $\mathrm{CD}_{2} \mathrm{Cl}_{2}$ at $253 \mathrm{~K}$.

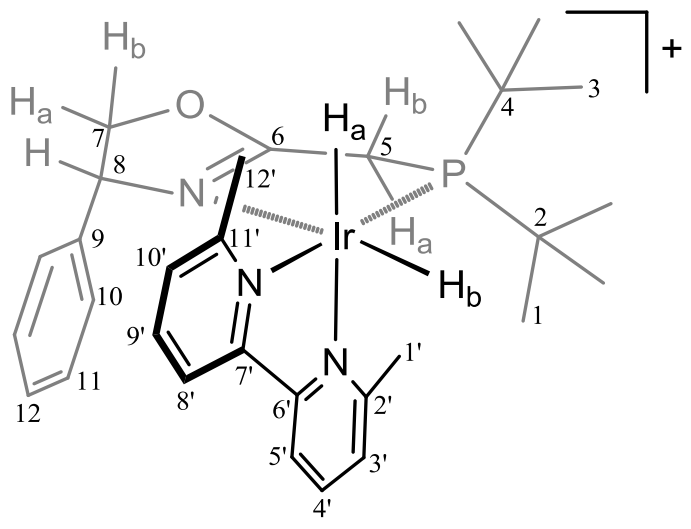

\begin{tabular}{ccc} 
Position & $\begin{array}{c}\delta\left({ }^{1} \mathrm{H}\right) \\
{[\mathrm{ppm}]}\end{array}$ & $\begin{array}{c}\delta\left({ }^{13} \mathrm{C}\right) \\
{[\mathrm{ppm}]}\end{array}$ \\
\hline $\mathrm{Ir}-\mathrm{H}_{\mathrm{a}}$ & -25.55 & - \\
$\mathrm{Ir}-\mathrm{H}_{\mathrm{b}}$ & -23.26 & - \\
1 & 1.16 & 29.1 \\
2 & - & 32.9 \\
3 & 1.30 & 27.6 \\
4 & - & 39.6 \\
$5 \mathrm{a}$ & 3.00 & 25.9 \\
$5 \mathrm{~b}$ & 3.08 & 25.9 \\
6 & - & 176.2 \\
$7 \mathrm{a}$ & 4.57 & 77.1 \\
$7 \mathrm{~b}$ & 4.98 & 77.1 \\
8 & 5.00 & 71.1 \\
9 & - & 136.6 \\
10 & 6.62 & 127.3 \\
11 & 6.50 & 127.7 \\
12 & 6.69 & 128.8 \\
\hline
\end{tabular}

\begin{tabular}{ccc} 
Position & $\begin{array}{c}\delta\left({ }^{1} \mathrm{H}\right) \\
{[\mathrm{ppm}]}\end{array}$ & $\begin{array}{c}\delta\left({ }^{13} \mathrm{C}\right) \\
{[\mathrm{ppm}]}\end{array}$ \\
\hline $1^{\prime}$ & 2.70 & 29.6 \\
$2^{\prime}$ & - & 160.5 \\
$3^{\prime}$ & 7.26 & 125.5 \\
$4^{\prime}$ & 7.69 & 138.2 \\
$5^{\prime}$ & 7.67 & 119.1 \\
$6^{\prime}$ & - & 158.1 \\
$7^{\prime}$ & - & 158.4 \\
$8^{\prime}$ & 7.64 & 120.3 \\
$9^{\prime}$ & 7.75 & 137.7 \\
$1^{\prime}$ & 7.42 & 125.9 \\
$1^{\prime}$ & - & 161.0 \\
$1^{\prime}$ & 2.91 & 31.6
\end{tabular}


Table S3. ${ }^{1} \mathrm{H}$ - and ${ }^{13} \mathrm{C}-\mathrm{NMR}$ data for the minor species (6b) of salt 6 in $\mathrm{CD}_{2} \mathrm{Cl}_{2}$ at $253 \mathrm{~K}$.

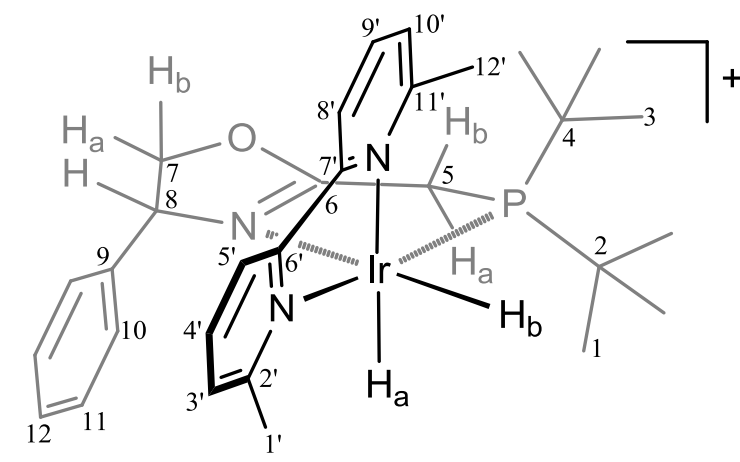

\begin{tabular}{ccc} 
Position & $\begin{array}{c}\delta\left({ }^{1} \mathrm{H}\right) \\
{[\mathrm{ppm}]}\end{array}$ & $\begin{array}{c}\delta\left({ }^{13} \mathrm{C}\right) \\
{[\mathrm{ppm}]}\end{array}$ \\
\hline $\mathrm{Ir}-\mathrm{H}_{\mathrm{a}}$ & -25.86 & - \\
$\mathrm{Ir}_{\mathrm{H}} \mathrm{H}_{\mathrm{b}}$ & -23.34 & - \\
1 & 1.31 & 27.9 \\
2 & - & 39.0 \\
3 & 1.16 & 29.0 \\
4 & - & 33.2 \\
$5 \mathrm{a}$ & 3.16 & 25.8 \\
$5 \mathrm{~b}$ & 2.91 & 25.8 \\
6 & - & 177.1 \\
$7 \mathrm{a}$ & 4.48 & 78.2 \\
$7 \mathrm{~b}$ & 4.80 & 78.2 \\
8 & 4.53 & 70.0 \\
9 & - & 138.9 \\
10 & 6.73 & 127.5 \\
11 & 7.18 & 129.5 \\
12 & 7.30 & 129.4 \\
\hline
\end{tabular}

\begin{tabular}{ccc} 
Position & $\begin{array}{c}\delta\left({ }^{1} \mathrm{H}\right) \\
{[\mathrm{ppm}]}\end{array}$ & $\begin{array}{c}\delta\left({ }^{13} \mathrm{C}\right) \\
{[\mathrm{ppm}]}\end{array}$ \\
\hline $1^{\prime}$ & 1.75 & 30.0 \\
$2^{\prime}$ & - & 162.8 \\
$3^{\prime}$ & 7.09 & 125.8 \\
$4^{\prime}$ & 7.68 & 137.8 \\
$5^{\prime}$ & 7.78 & 120.5 \\
$6^{\prime}$ & - & 158.2 \\
$7^{\prime}$ & - & 159.1 \\
$8^{\prime}$ & 7.93 & 119.3 \\
$9^{\prime}$ & 7.87 & 139.2 \\
$10^{\prime}$ & 7.39 & 125.9 \\
$11^{\prime}$ & - & 161.5 \\
$12^{\prime}$ & 2.68 & 28.2
\end{tabular}


Table S4. Crystal data for complex 1.

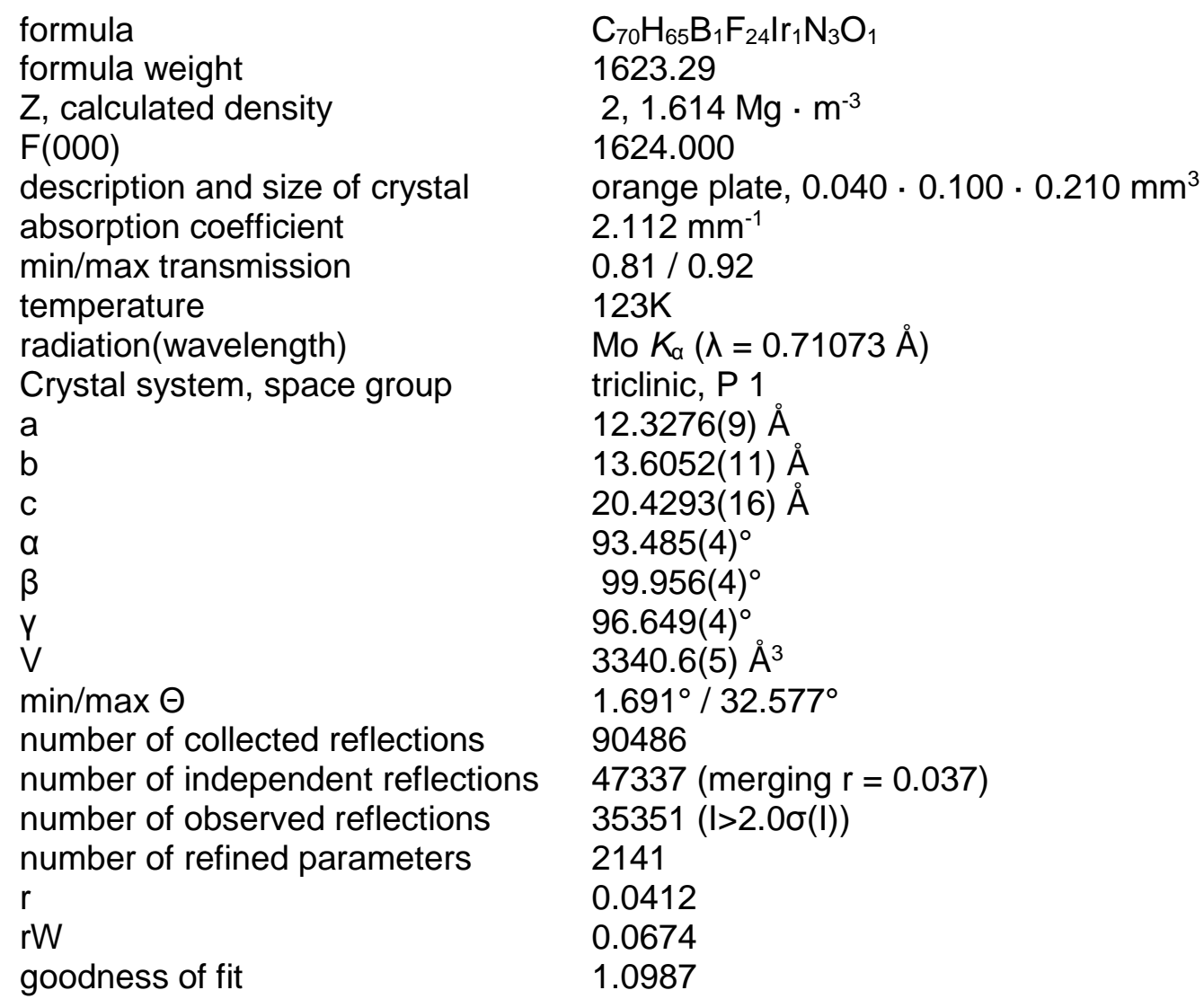

Table S5. Crystal data for complex 2.

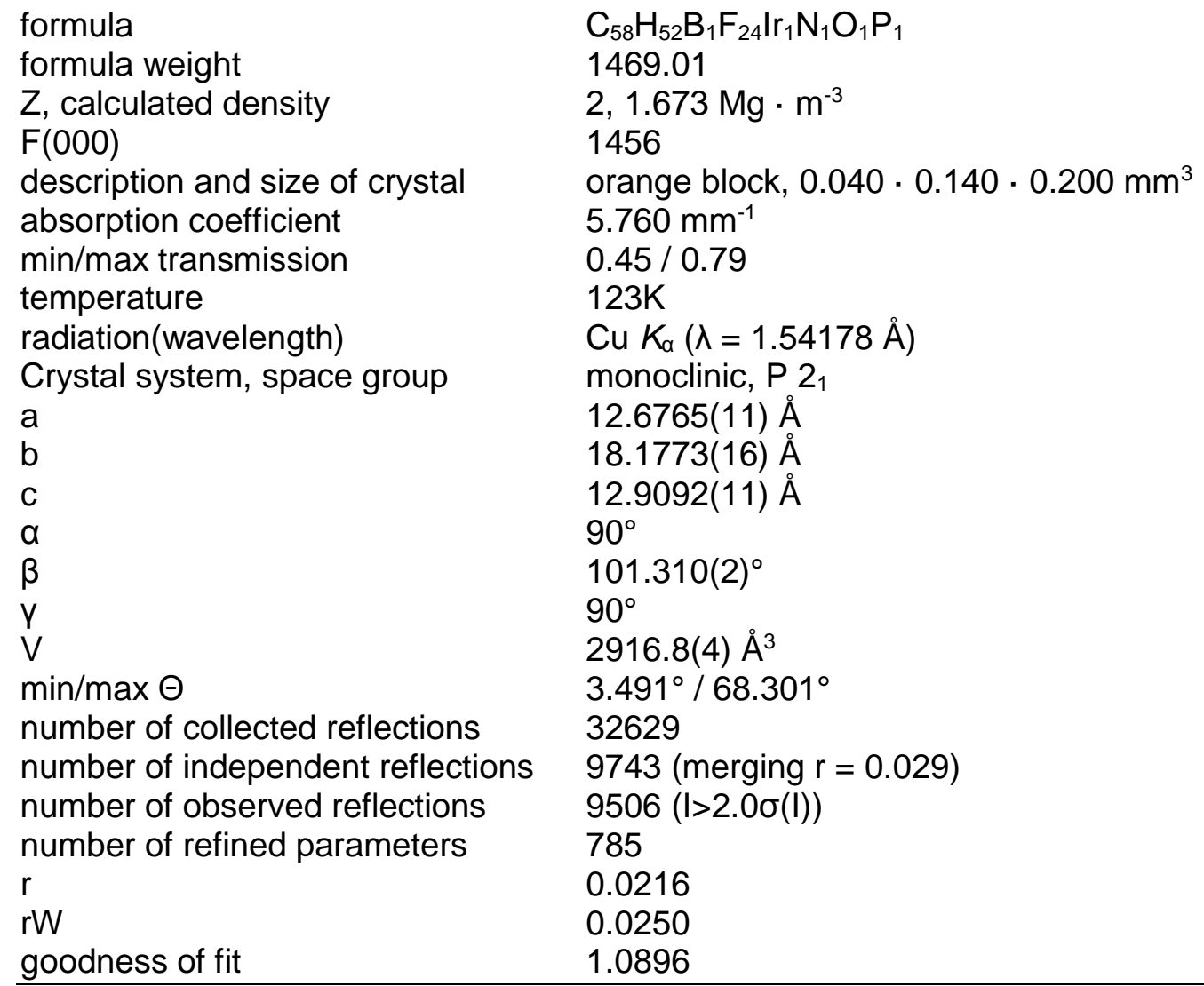


Table S6. Crystal data for complex 5.

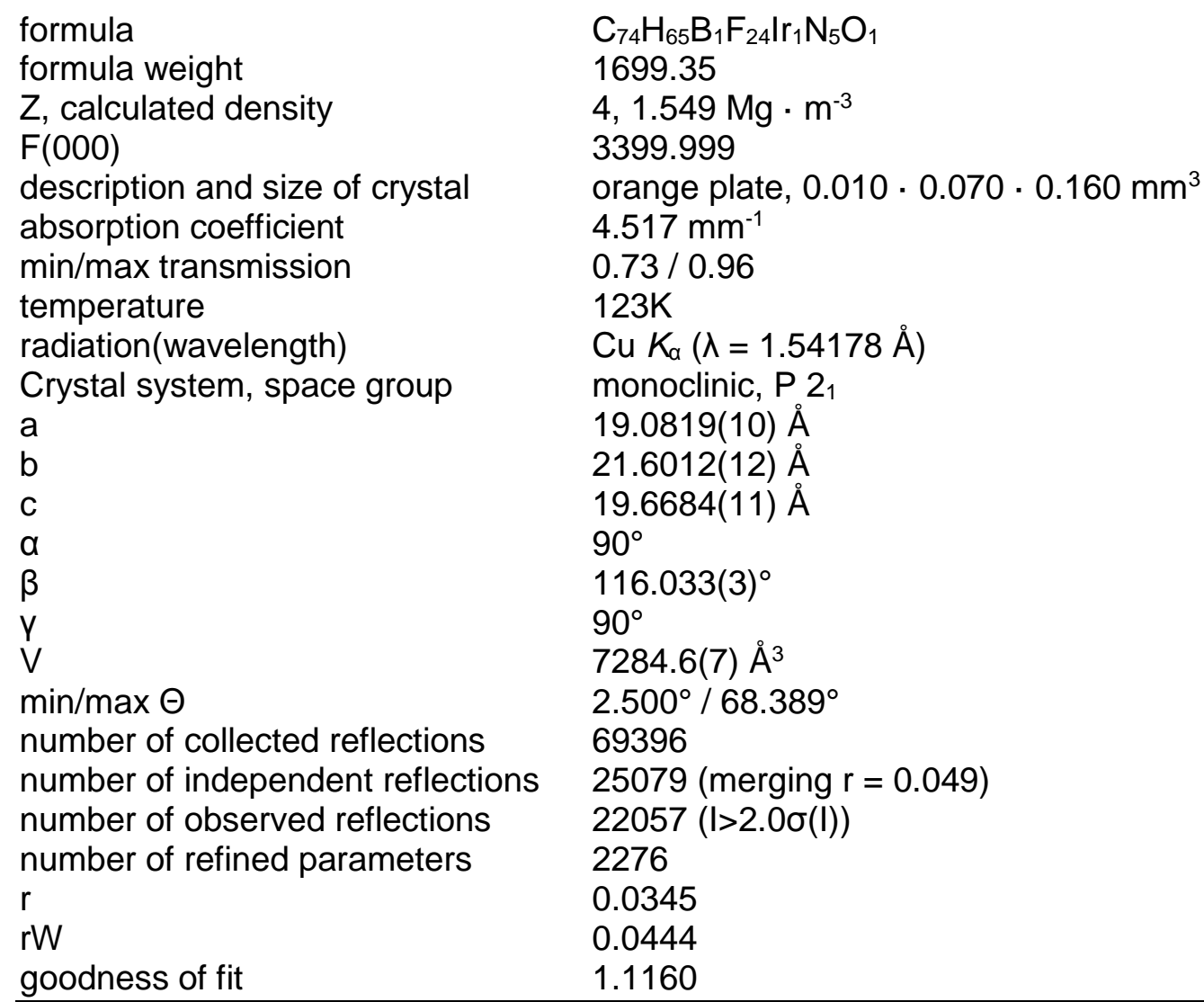

Table S7. Crystal data for complex $6 \mathbf{a}$.

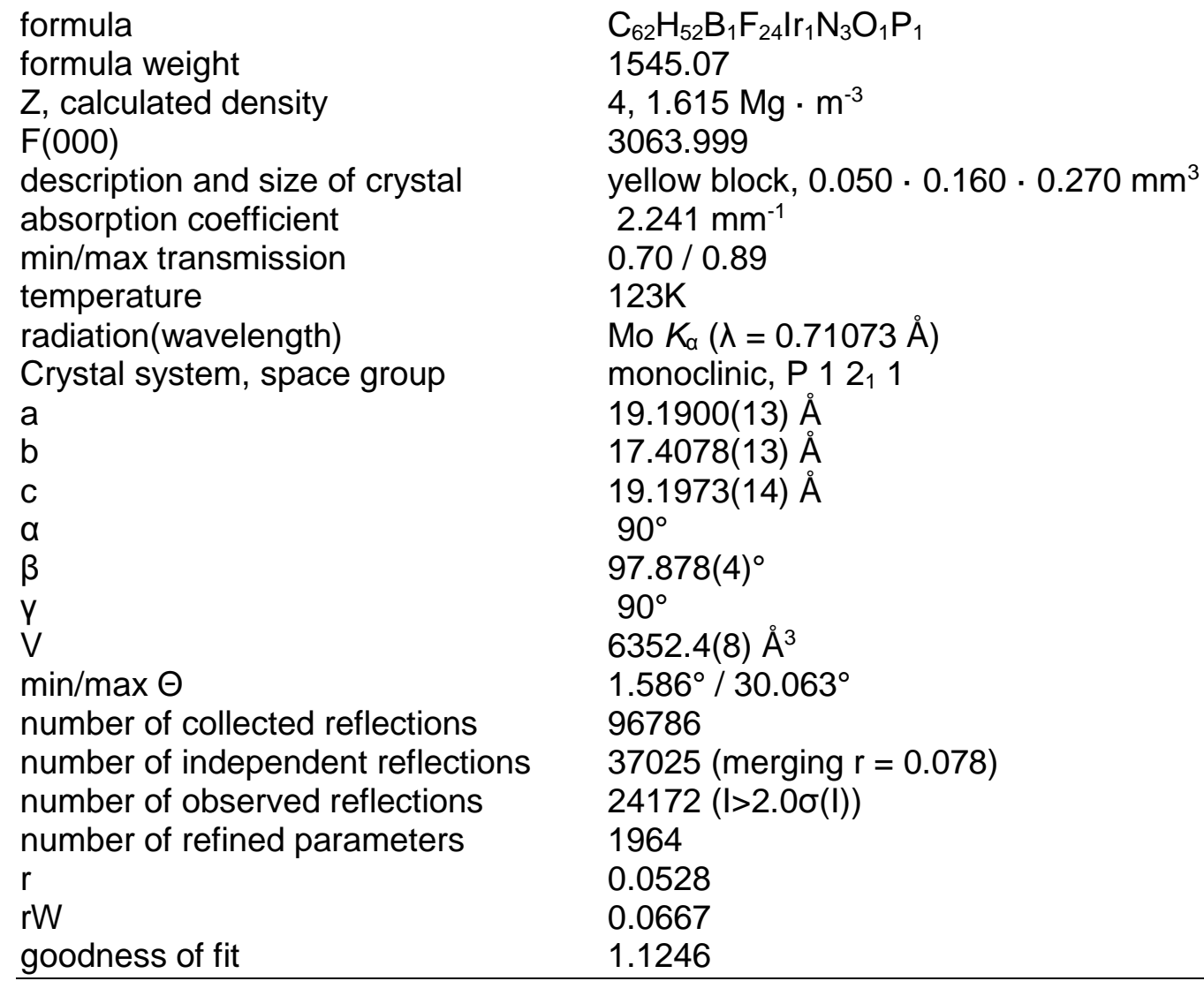

goodness of fit 


\section{Spectra for complex 5 at $295 \mathrm{~K}$.}

$500 \mathrm{MHz} 1 \mathrm{H}$ NMR spectrum of complex 5

命 万゙

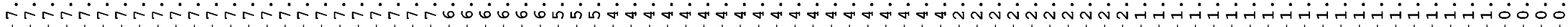
(1)

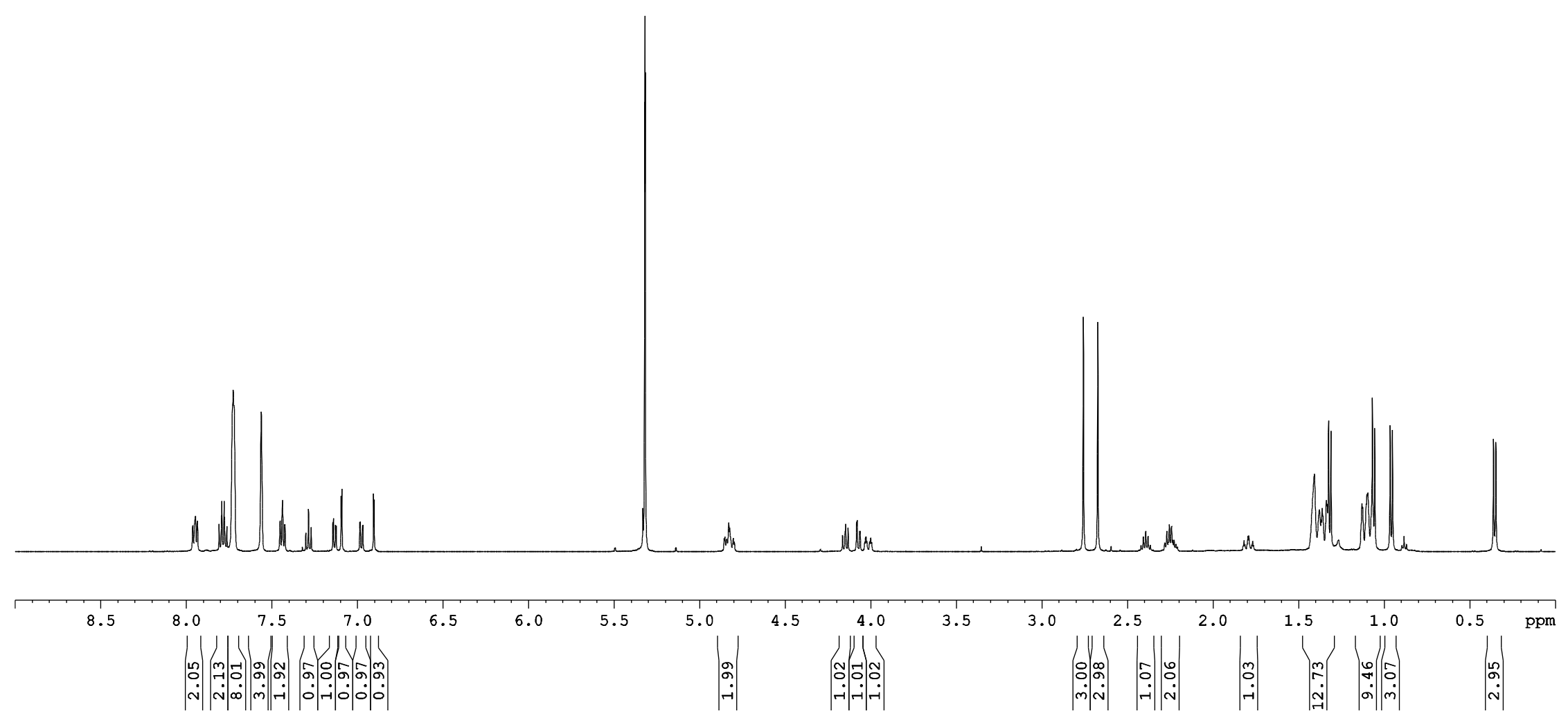


$500 \mathrm{MHz}$ 1H NMR spectrum of complex $\mathbf{5}$ (hydride region)

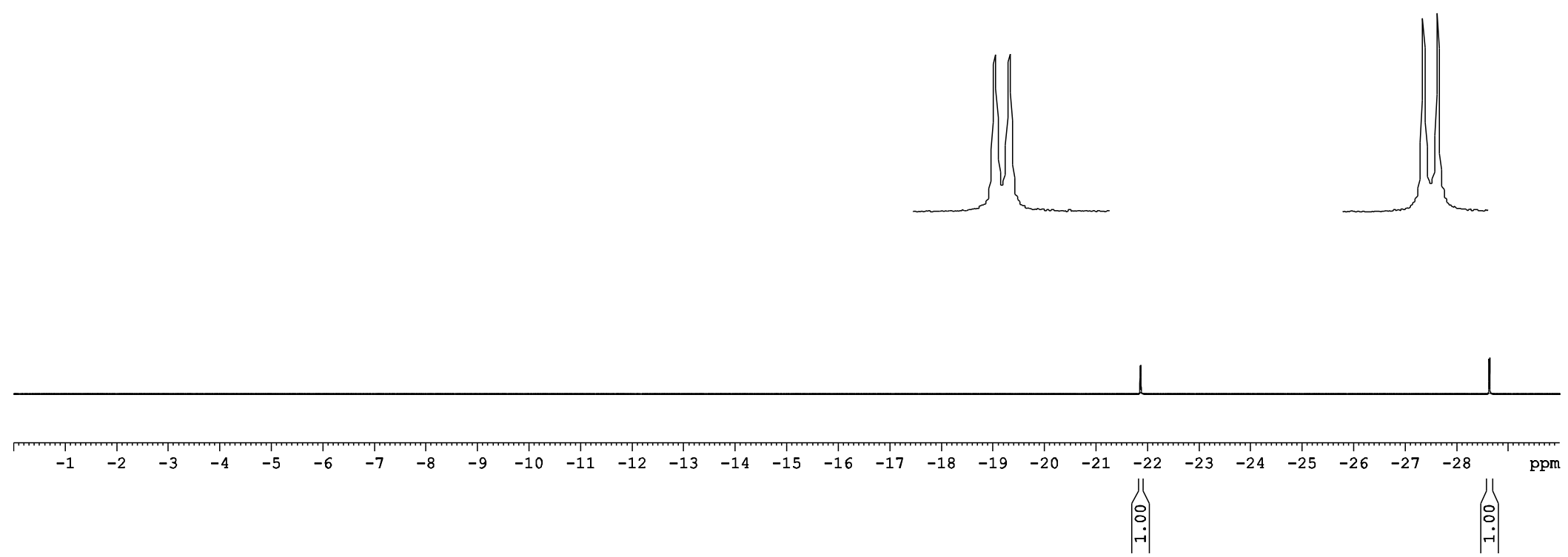




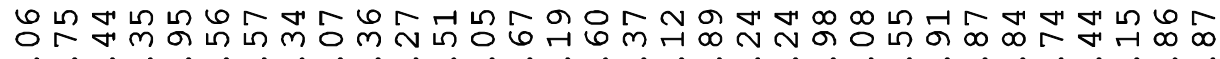

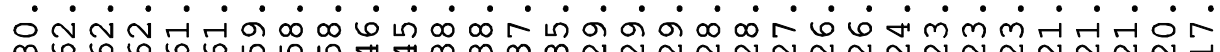

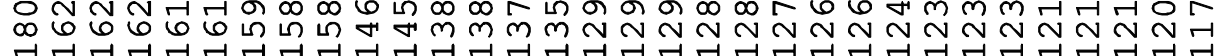

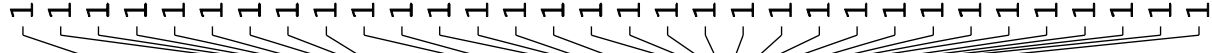

No

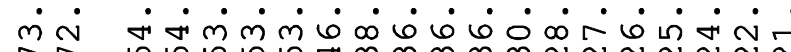

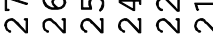

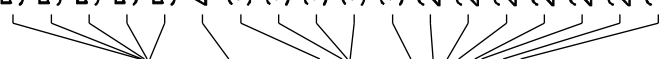

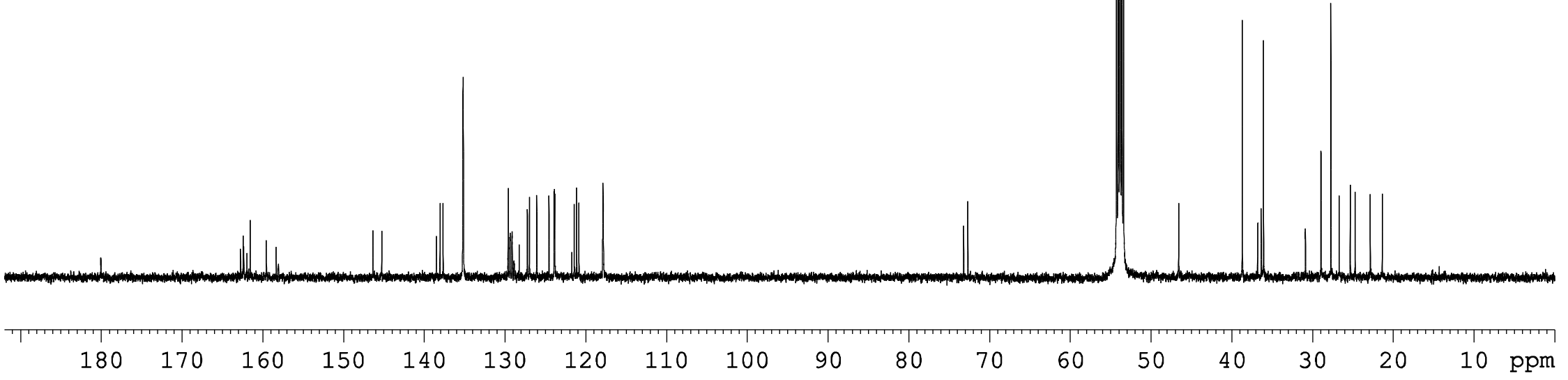




\section{Spectra for complex 6 at $253 \mathrm{~K}$.}

$500 \mathrm{MHz} 1 \mathrm{H}$ NMR spectrum of complex 6 (isomeric mixture of $\mathbf{6 a}$ and $\mathbf{6 b}$ )

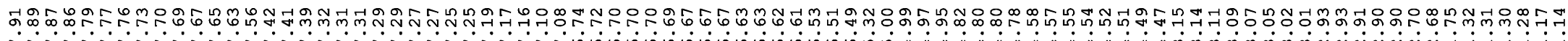

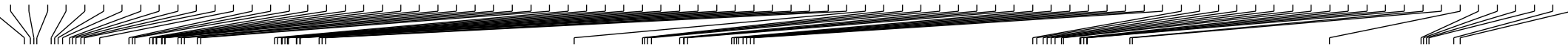

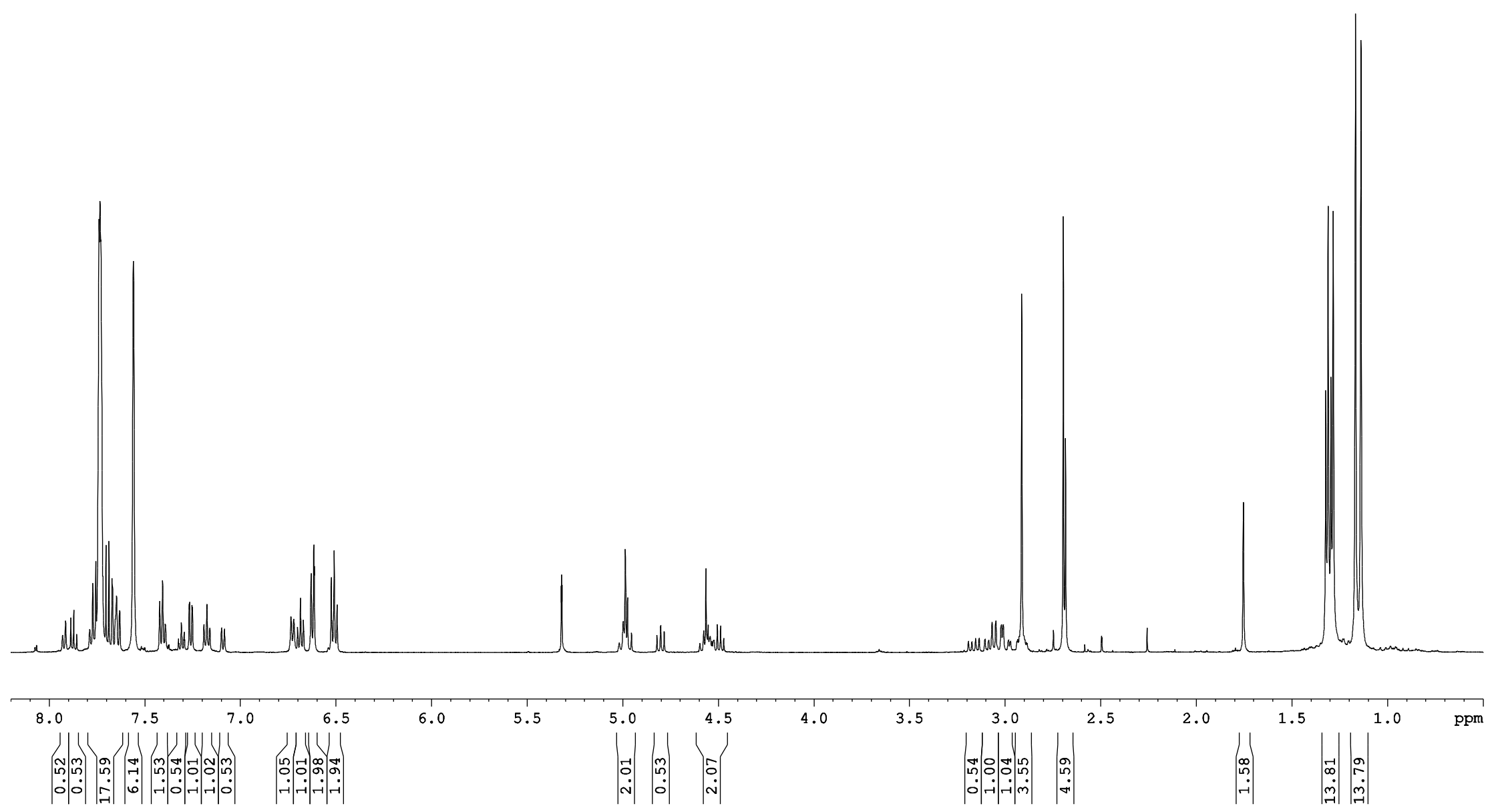




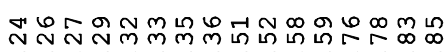

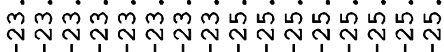
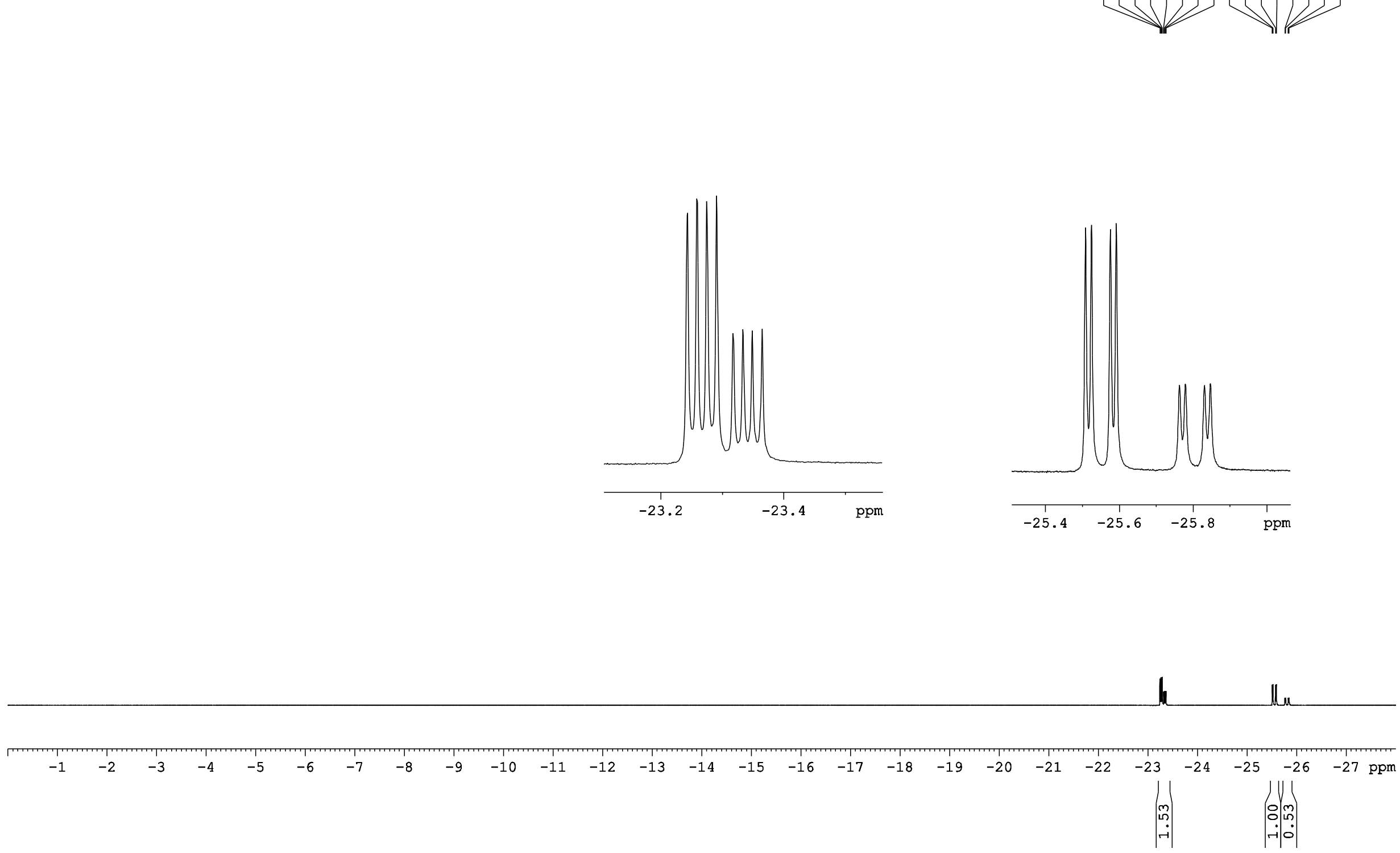

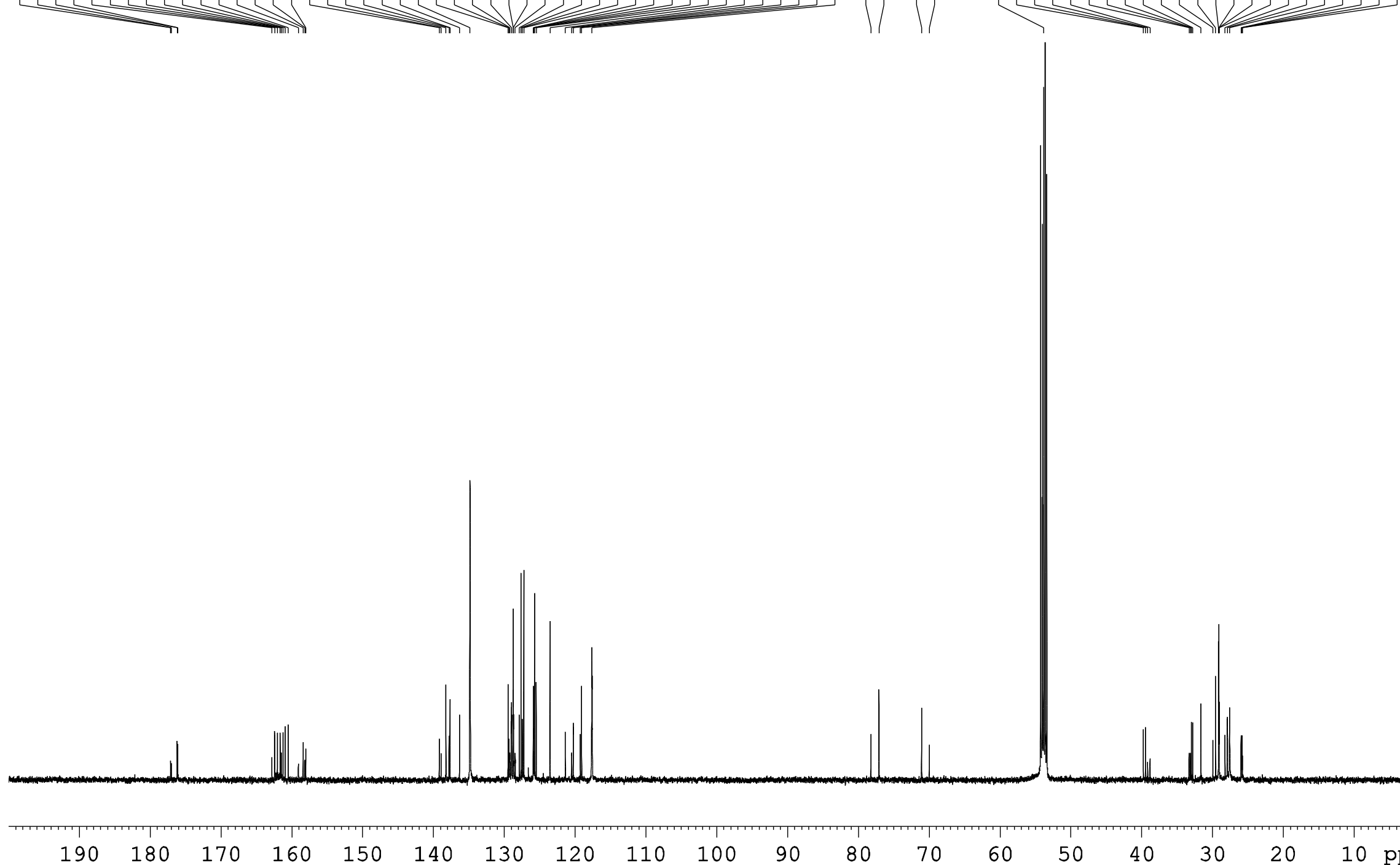

190180

160

150

140

130

120

S12 
$202 \mathrm{MHz} 31 \mathrm{P}\{1 \mathrm{H}\}$ NMR spectrum of complex $\mathbf{6}$ (isomeric mixture of $\mathbf{6 a}$ and $\mathbf{6} \mathbf{b}$ )

|

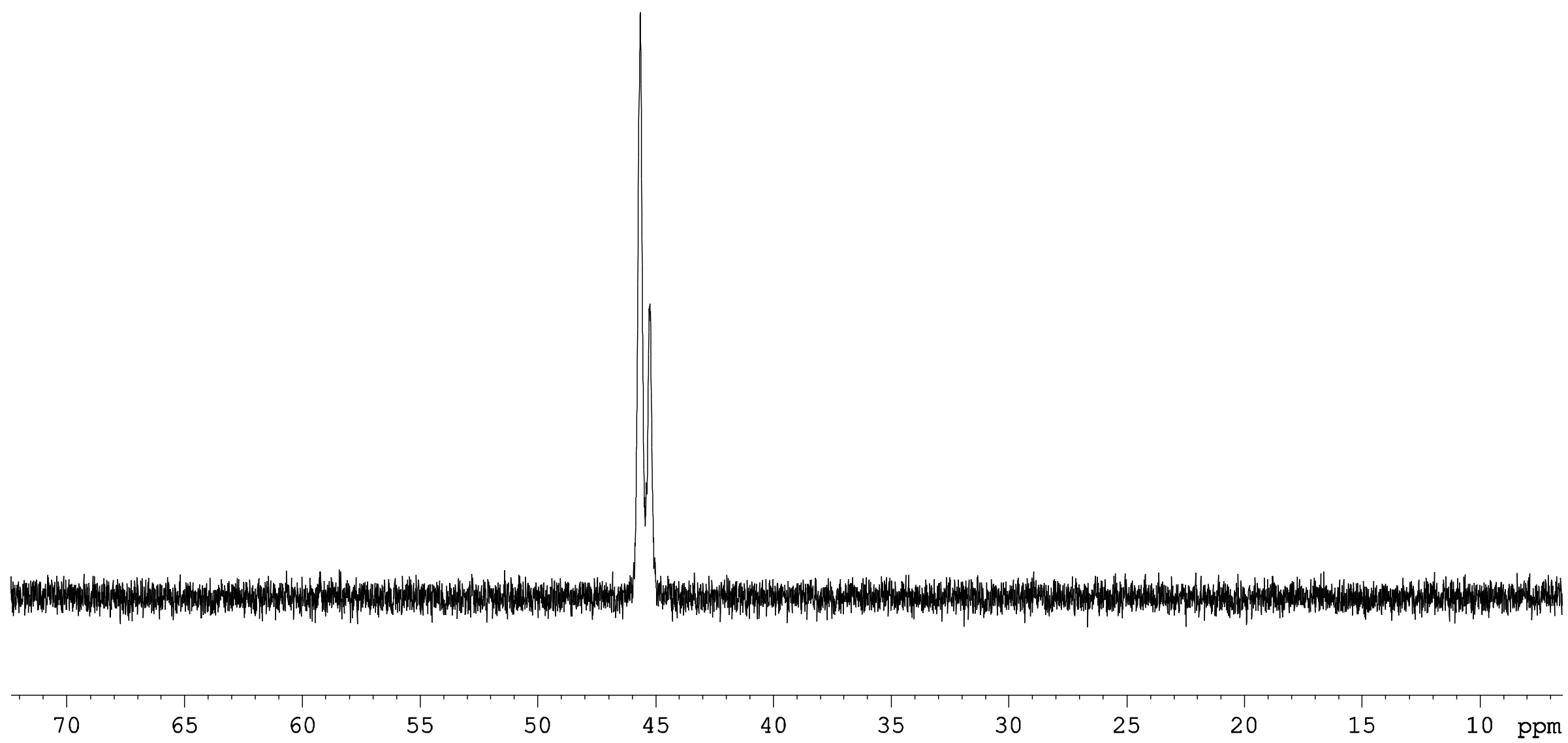

